\title{
Perfil clínico das internações por herpes zoster em um hospital universitário
}

\author{
Túlio G. M. C. Júnior ${ }^{1 *}$ Valesca L. Santos, ${ }^{1}$ Cintia M. Martins,,${ }^{1}$ Ana C. B. Miranda, ${ }^{1}$ Raíssa P. Nunes, ${ }^{1}$ \\ Esther B. Palitot ${ }^{2}$
}

\begin{abstract}
Resumo
Introdução: Herpes zoster (HZ) é uma doença infecciosa relativamente comum causada pela reativação do vírus varicela zoster (VVZ) nos nervos cranianos e nos gânglios das raízes espinhais dorsais. Apesar de um aumento na prevalência nos últimos anos e da elevada morbidade, ainda há poucos estudos epidemiológicos sobre a doença. Objetivo: Conhecer e analisar o perfil clínico dos hospitalizados por HZ. Materiais e métodos: Foram analisados todos os prontuários dos internados por HZ, entre 2009 e 2015, em um Serviço de Doenças Infectocontagiosas de um Hospital Universitário, observando aspectos como sexo, idade, tempo de internação e possíveis intercorrências. Resultados: Do total da amostra, 50\% eram do sexo feminino; a faixa etária mais acometida foi de até 20 anos, representada por 33,33\% dos pacientes, e o local da manifestação das lesões foi na face em $56,6 \%$ dos casos. A maioria $(48,89 \%)$ ficou hospitalizada entre 1 e 2 semanas, tendo como principais intercorrências as infecções secundárias, presentes em 40\% dos pacientes. Conclusões: Reafirma-se as morbidades que essa infecção traz para os indivíduos, ônus das prolongadas internações e os custos para o sistema de saúde, evidenciando-se a necessidade de estratégias eficazes de prevenção da doença e suporte aos acometidos.
\end{abstract}

Descritores: Herpes zoster; Herpesviridae; Hospitalização.

\section{Abstract \\ Clinical profile of herpes zoster hospitalizations at a university hospital}

Introduction: Herpes zoster (HZ) is a relatively common infectious disease caused by the reactivation of the varicella zoster virus (VZV) in the cranial nerves and in the dorsal spinal root ganglia. Although prevalence has increased in recent years and despite the high morbidity, there are still few epidemiological studies on the disease. Objective: To know and to analyze the clinical profile of hospitalized patients by HZ. Materials and methods: All patients' records of HZ hospitalizations were analyzed between 2009 and 2015 in an Infectious Diseases Service of a University Hospital, observing aspects such as gender, age, length of hospital stay and possible intercurrences. Results: Of the total sample, 50.0\% were female and 50.0\% were male; the most affected age group was up to 20 years old, represented by $33.33 \%$ of the patients, with $56.6 \%$ of the lesions manifested in the face. The majority of them, $48.89 \%$, were hospitalized between 1 and 2 weeks, and the main complications were secondary infections, present in $40 \%$ of the patients. There was no predilection for sex; the majority of
1. Universidade Federal da Paraíba. João Pessoa, PB, Brasil.

2. Departamento de Promoção à Saúde. Universidade Federal da Paraíba. João Pessoa, PB, Brasil.

*Endereço para correspondência:

Rua Avelino Cunha, no 125

João Pessoa, Paraíba, Brasil. CEP: 58020-650.

E-mail: t.germano.93@gmail.com

Revista HUPE, Rio de Janeiro, 2017;16(2):85-88

doi: $10.12957 /$ rhupe.2017.37633

Recebido em 30/04/2018. Aprovado em 13/06/2018.

whom were young or elderly adults, hospitalized between 1 and 2 weeks, having as main intercurrence secondary infections. Conclusions: The morbidities that this infection brings to individuals, the burden of prolonged hospitalizations and the costs to the health system are reaffirmed, thus making clear the need for effective strategies for disease prevention and support to the affected.

Key words: Herpes zoster; Herpesviridae; Hospitalization.

\section{Resumen}

\section{Perfil clinico de las internaciones por herpes zoster en un hospital universitario}

Introducción: Herpes zoster (HZ) es una enfermedad infecciosa relativamente común causada por la reactivación del virus varicela zoster (VVZ) en los nervios craneales y en los ganglios de las raíces espinales dorsales. Aunque la prevalencia ha crecido en los últimos años y a pesar de la alta morbilidad, todavía hay pocos estudios epidemiológicos sobre la enfermedad. Objetivo: Conocer y analizar el perfil clínico de los hospitalizados por HZ. Material y métodos: Se analizaron todos los prontuarios de los internados por HZ, entre 2009 y 2015, en un Servicio de Enfermedades Infectocontagiosas de un Hospital Universitario, observando aspectos como sexo, edad, tiempo de internación y posibles intercurrencias. Resultados: El 50,0\% eran del sexo femenino y el 50,0\% del sexo masculino; el grupo de edad más acometida fue de hasta los 20 años, representada por el 33,33\% de los pacientes, siendo $56,6 \%$ de las lesiones manifestadas en la cara. La mayoría, 48,89\%, quedó hospitalizada entre 1 y 2 semanas, teniendo como principales intercurrencias las infecciones secundarias, presentes en el $40 \%$ de los pacientes. No hubo predilección por sexo; la mayoría eran adultos jóvenes 


\section{Artigo original}

o ancianos, y que quedaron internados entre 1 y 2 semanas, teniendo como principal intercurrencia las infecciones secundarias. Conclusiones: Se reafirma, así, las morbilidades que esta infección trae para los individuos, la carga de las prolongadas internaciones y los costos para el sistema de salud, tornándose

\section{Introdução}

Herpes zoster (HZ) é uma doença infecciosa pela reativação do vírus varicela zóster (VZV), que permanece latente nos gânglios das raízes dorsais, nos gânglios autonômicos e nos nervos cranianos após uma infecção primária (varicela), sendo caracterizada uma erupção localizada com vesículas, além de dor e inflamação no trajeto nervoso afetado., ${ }^{1,2}$

Constitui-se como doença infecciosa comum, dado que em média um terço da população já apresentou o quadro, sendo a incidência maior em faixas etárias avançadas, visto que há um declínio na imunidade celular com o passar do tempo. ${ }^{3}$

A doença pode evoluir para a cura em poucas semanas ou a dor pode continuar por meses ou anos. ${ }^{4,5} \mathrm{~A}$ persistência da dor após a cura da erupção é a complicação mais frequente, sendo denominada de neuralgia pós-herpética (NPH). Esta é uma importante causa de grandes incapacidades físicas e problemas emocionais, o que incapacita o paciente - sobretudo os idosos, que representam o grupo mais acometido - de realizar atividades diárias. Aproximadamente metade dos pacientes com idade superior a 70 anos desenvolvem $\mathrm{NPH}^{6,7}$

Além desta, também há a possibilidade de complicações oftálmicas, paralisia de nervos cranianos e periféricos, paresia segmentar, somadas as infecções bacterianas secundárias. ${ }^{8}$ Casos graves destas complicações muitas vezes necessitam de hospitalização.,99

Apesar de um aumento na prevalência nos últimos anos, ainda há uma relativa carência de análise do perfil clínico dos acometidos por herpes zoster, uma doença com elevada morbidade e grande importância no contexto da saúde pública. Nesse sentido, o objetivo é realizar um estudo com base nas internações por zoster em um Serviço de Doenças infectocontagiosas de um Hospital Universitário.

\section{Materiais e métodos}

Trata-se de uma pesquisa de carácter retrospectivo e observacional. O projeto foi aprovado pelo Comitê de Ética em Pesquisa (CEP) do Hospital Universitário. Foram analisados 90 prontuários de pacientes internados por HZ entre o período de agosto de 2009 a clara la necesidad de estrategias eficaces de prevención de la enfermedad y soporte a los acometidos.

Palabras clave: Herpes zoster; Herpesviridae; Hospitalización.

agosto de 2015, avaliando as seguintes variáveis: sexo, idade, tempo de internação, local das lesões e possíveis intercorrências.

Os dados encontrados foram registrados e organizados em forma de gráficos e tabelas pelo programa Microsoft Office Excel Professional Plus 2013. Posteriormente, as informações analisadas foram comparadas com artigos da literatura científica.

\section{Resultados}

A prevalência quanto ao sexo, idade, além do tempo de internação e presença de infecção pelo vírus HIV, podem ser observadas pela tabela abaixo (Tabela 1):

É importante ressaltar que em todos os casos os internados apresentaram quadro de varicela previamente ao HZ, principalmente na infância e na juventude. No entanto, não constava nos prontuários dos pacientes a história detalhada do contágio.

Quanto ao local das lesões, a face foi a região mais frequente, correspondendo a 51 pacientes $(56,6 \%)$, tendo a seguinte distribuição quanto ao trajeto nervoso: 40 indivíduos $(78,4 \%)$ com o ramo oftálmico do trigêmeo acometido, 7 (13,7\%) no segmento maxilar e/ou mandibular e 4 (7,8\%) no nervo facial. A segunda área mais acometida foi a região torácica, com 22 casos $(24,4 \%)$ e em seguida a região dorsal, correspondente a 18 casos (20\%).

Observou-se ainda acometimento do sistema nervoso, sendo 5 casos $(5,5 \%)$ de NPH, 2 (2,2\%) de paralisia facial e $1(1,1 \%)$ de Síndrome de Ramsey Hunt. Além disso, 36 pacientes (40\%) apresentaram algum tipo de infecção durante o decorrer da internação, dentre eles, 29 casos de etiologia bacteriana $(80,5 \%)$.

\section{Discussão}

Sabe-se que o HZ é uma doença relativamente frequente na população, acometendo até $30 \%$ dos indivíduos, durante alguma fase de sua vida. Desse total, até 3\% necessitam de internação. ${ }^{7,9}$ Alguns estudos mostram que a taxa de hospitalização varia de 2 a 25/100.000 pessoas-ano e os custos para manter uma paciente com zoster internado em um hospital são altos. ${ }^{5}$ 
Tabela 1. Características dos pacientes internados por herpes zoster $(n=90), X, 2016$

\begin{tabular}{|c|c|c|}
\hline Variável & $\begin{array}{l}\text { Frequência } \\
\text { absoluta (n) }\end{array}$ & $\begin{array}{l}\text { Frequência } \\
\text { relativa (\%) }\end{array}$ \\
\hline \multicolumn{3}{|l|}{ Sexo } \\
\hline Masculino & 45 & 50,00 \\
\hline Feminino & 45 & 50,00 \\
\hline \multicolumn{3}{|l|}{ Faixa etária } \\
\hline Abaixo de 18 anos & 29 & 32,22 \\
\hline $18-29$ anos & 7 & 7,77 \\
\hline $30-39$ anos & 4 & 4,44 \\
\hline $40-49$ anos & 12 & 13,3 \\
\hline $50-59$ anos & 7 & 7,77 \\
\hline A partir de 60 anos & 31 & 34,44 \\
\hline \multicolumn{3}{|l|}{ Tempo de internação } \\
\hline 0-1 Semana & 26 & 28,88 \\
\hline 1-2 Semanas & 45 & 50 \\
\hline 2-3 semanas & 16 & 17,77 \\
\hline 3-4 semanas & 1 & 1,11 \\
\hline 4-5 semanas & 1 & 1,11 \\
\hline Mais de 5 semanas & 1 & 1,11 \\
\hline \multicolumn{3}{|l|}{ HIV } \\
\hline Reagente & 4 & 4,44 \\
\hline Não reagente & 86 & 95,66 \\
\hline Total & 90 & 100 \\
\hline
\end{tabular}

O tempo de internação hospital gira em torno de 6,4 a 13,3 dias. $^{2}$ No presente estudo os pacientes permaneceram internados na sua maioria por volta de 7 a 14 dias.

Não houve diferença da prevalência do herpes zoster quanto ao sexo. Os estudos epidemiológicos internacionais mostram disparidades em relação à prevalência dessa enfermidade entre os sexos - que podem ocorrer por diferenças no processo de amostragem ou pelo fato de homens procurarem menos o serviço de saúde do que as mulheres. Acredita-se, portanto, que não há predileção sexual no HZ. ${ }^{8}$

Tais estudos também revelam que o acometimento aumenta com a progressão da idade, sendo esse o fator de risco mais importante para a doença. Há o aumento significativo da infecção após os 50 anos de idade, sendo importante ressaltar que após os 80 anos cerca de 50\% das pessoas irão apresentar a doença. 5,7, 9,10

Essa pesquisa mostrou que dentre os internados por HZ houve dois picos de maior incidência da faixa etária, que foram os indivíduos de até 18 anos e os acima de 60 anos, correspondendo respectivamente a 29 indivíduos $(32,22 \%)$ e 31 (34,44\%).

Sabe-se que essa infecção está diretamente relacionada com a imunidade e que pessoas imunocomprometidas apresentam maior propensão a desenvolver este tipo de enfermidade. O controle da reativação de VZV depende da manutenção de níveis adequados de imunidade celular-mediada para o VZV. $., 7,11$ Nesse sentido, a infecção pelo HIV é um fator de risco para o desenvolvimento do herpes zoster (HZ) e suas complicações. Antes da terapia antirretroviral (TARV) a incidência de HZ nos infectados pelo HIV variava de 2,9 a 5.1/100 pessoas-ano. Estudos de coorte posteriores sugerem uma diminuição da incidência, porém permanecendo maior do que na população geral. ${ }^{12}$ Nesta pesquisa, registrou-se uma concomitância dessas infecções em 4 pacientes $(4,44 \%)$ dos hospitalizados.

As lesões aparecem primeiramente como pápulas eritematosas que evoluem para vesículas sob base eritematosa e edematosa, localizadas em um ou mais dermátomos contíguos unilateralmente. Os trajetos mais acometidos são: torácico (53\%), cervical (20\%) e trigeminal (15\%), incluindo o ramo oftálmico e lombossacral (11\%). ${ }^{2}$ Analisando os indivíduos da amostra, observa-se que a região mais acometida da face, correspondente ao trajeto do nervo trigêmeo, destacando-se o ramo oftálmico, seguida da região do tórax e dorso.

Uma complicação relativamente comum é a neuralgia pós-herpética (PHN), operacionalmente definida como a dor com duração superior a 90 dias após a erupção cutânea, outras afecções são doenças neurológicas como meningoencefalite, mielite e doença vascular, incluindo artrite temporal VZV e oculares (necrose da retina externa aguda ou progressiva). ${ }^{4,9}$ No presente estudo as complicações mais frequentemente encontradas foram as infecciosas e em segundo lugar encontram-se as neurológicas.

\section{Conclusão}

Evidencia-se que dentro do grupo estudado não houve predileção por sexo; a maioria era formada por adultos jovens e idosos, cujas lesões predominavam em face, e que ficaram internados entre 1 e 2 semanas, tendo como principais intercorrências as infecções secundárias.

Conclui-se que embora na maioria dos casos ocorra 


\section{Artigo original}

cura em poucas semanas, o quadro clínico pode evoluir com manifestações graves e complicações, prolongando o tempo de internação, gerando mais morbidades e maiores custos ao sistema de saúde.

Diante desse cenário, é importante conhecer o perfil dos pacientes diagnosticados com herpes zoster devido às complicações da doença, que podem causar grande impacto na qualidade de vida, para que assim, ações de promoção e intervenção de saúde, como a vacinação contra o zoster, hoje apenas disponível na rede privada, possam ser mais bem avaliadas.

\section{Referências}

1. Muñoz-Quiles C, López-Lacort M, Orrico-Sánchez A, et al. Impact of postherpetic neuralgia: A six year population-based analysis on people aged 50 years or older. J Infect [Internet]. Elsevier Ltd; 2018;0:1-6. Available from: https://doi. org/10.1016/j.jinf.2018.04.004

2. Vázquez M, Cravioto P, Galván F, et al. Varicela y herpes zóster: retos para la salud pública. Salud Publica Mex. 2017;59(6):650-6.

3. Turner NM, Macrae J, Nowlan ML, et al. Quantifying the incidence and burden of herpes zoster in New Zealand general practice : a retrospective cohort study using a natural language processing software inference algorithm. BMJ Open 2018. 2018;8:1-9.

4. Portella AVT, Souza LCB, Gomes JMA. Herpes-zoster and post-herpetic neuralgia. Rev Dor. 2013; 14(3): 210-5.

5. Kawai K, Gebremeskel BG, Acosta CJ. Systematic review of incidence and complications of herpes zoster: towards a global perspective. BMJ Open. 2014;4(6).

6. Gershon AA, Gershon MD. Pathogenesis and Current Approaches to Control of Varicella-Zoster Virus Infections. Clin Microbiol Rev. 2013;26(4):728-43.

7. Cohen J. Herpes Zoster. N Engl J Med. 2013;369(3):255-263.

8. Koshy E, Mengting L, Kumar H, et al. Epidemiology, treatment and prevention of herpes zoster: A comprehensive review. IJDVL. 2018;84(3):251-62.

9. Yawn BP, Gilden D. The global epidemiology of herpes zoster. Neurology. 2013;81(10):928-930.

10. Pinchinat $\mathrm{S}$, Cebrián-cuenca AM, Bricout $\mathrm{H}$, et al. Similar herpes zoster incidence across Europe: results from a systematic literature review. BMC Infectious Diseases. 2013;13(1).

11. Chen S, Suaya J, Li Q, et al. Incidence of herpes zoster in patients with altered immune function. Infection. 2014;42(2):325-334.

12. Jansen K, Haastert B, Michalik $C$, et al. Incidence and risk factors of herpes zoster among hiv-positive patients in the german competence network for HIV/AIDS (KompNet): a cohort study analysis. BMC Infect Dis. 2013;13:372. 